\title{
SANS Study of Liquid-Liquid Phase Transition in Protein Electrolyte Solution
}

\author{
Akshay J. Chinchalikar $^{1 *}$, V. K. Aswal ${ }^{1}$, J. Kohlbrecher ${ }^{2}$, A. G. Wagh ${ }^{1}$ \\ ${ }^{1}$ Solid State Physics Division, Bhabha Atomic Research Centre, Mumbai-400085, India \\ ${ }^{2}$ Laboratory for Neutron Scattering, ETH Zurich \& Paul Scherrer Institut, CH-5232 PSI Villigen, Switzerland \\ *E-mail:akshayc@barc.gov.in
}

\begin{abstract}
Small-angle Neutron Scattering (SANS) measurements have been performed on lysozyme protein solution to examine liquid-liquid phase transition with the addition of $\mathrm{NaCl}$. We show that the liquid-liquid phase transition is governed by the increase in the attractive interaction between protein molecules as tuned by the salt concentration. This attractive interaction is modeled by the Baxter's sticky hard sphere potential. It is found that when the attractive potential becomes significantly larger than the thermal energy protein molecules coalesce to form gel.
\end{abstract}

Keywords: lysozyme, small-angle neutron scattering, short range attraction, sticky hard sphere potential PACS: 82.70.Dd, 87.14E-, 87.15km

\section{INTRODUCTION}

The structural modifications in any colloidal system are an account of the interactional changes amongst the various constituents. Proteins are one of the important macromolecules and are part of most of the biological activities in living organisms. The protein interactions, by the virtue of complexity in their structures have been one of the challenging problem since last few years [1]. The interactional changes give rise to different phases like gel, cloud, crystal etc. To control different phases by tuning the interactions in protein solutions are of great interest for many applications in pharmaceutical, food and cosmetic industries. Proteins in general possess net charge in the solution which maintains the stability. It is believed that protein interactions can be described by the combination of short range attraction and long range repulsion [2]. The stability in protein solutions can be tuned by the addition of salts to examine different phases of protein solutions. Small-angle neutron scattering (SANS) is an ideal technique to study structure as well as interactions in these systems [3]. Herein, we report SANS studies of lysozyme protein solutions on addition of salt resulting in liquidliquid phase transitions to form gel.

\section{EXPERIMENTS}

Hen egg white lysozyme was obtained from FLUKA and used as obtained. All the samples were prepared in phosphate buffer having $\mathrm{pH}$ of 7.0. $\mathrm{D}_{2} \mathrm{O}$ was used as solvent instead of $\mathrm{H}_{2} \mathrm{O}$ for getting better contrast in SANS experiments. The measurements were performed on 5 wt $\%$ aqueous lysozyme solutions with varying concentration of $\mathrm{NaCl}$ in the range 0 to $0.6 \mathrm{M}$. The sample shows the gelation at $0.61 \mathrm{M} \mathrm{NaCl}$. Experiments were carried out at SANS-I facility, Swiss Spallation Neutron Source, SINQ, Switzerland. The mean wavelength of neutron beam used was $6 \AA$ and the data were collected in the wave vector transfer $Q$ range of 0.01 to $0.2 \AA^{-1}$. All the data were fitted by using non-linear least square fitting program.

\section{DATA ANALYSIS}

In SANS, one measures differential scattering cross section per unit volume $(d \Sigma / d \Omega)$ as a function of $Q$ and for a protein solution it can be written as

$$
\frac{d \sum}{d \Omega}(Q)=n\left(\rho_{p}-\rho_{s}\right)^{2} V_{p}^{2} P(Q) S(Q)
$$

where $n$ is the number density of macromolecules, $\rho_{p}$ and $\rho_{s}$ are scattering length densities of protein molecule and solvent, respectively. $P(Q)$ is intraparticle structure factor which contains information about shape and size of particle. $S(Q)$ represents interparticle structure factor and influenced by the interaction between particles. $P(Q)$ has been calculated for prolate ellipsoidal shape of protein molecules and $S(Q)$ by the Baxter's sticky hard sphere attraction model [4]. 


\section{RESULTS AND DISCUSSIONS}

Figure 1 shows SANS data of 5 wt \% lysozyme solution with varying concentration of $\mathrm{NaCl}$. The pure lysozyme data suggest a stable system and is fitted with the screened Coulomb repulsive potential [5]. The protein-protein correlation peak is observed at 0.045 $\AA^{-1}$. On addition of salt, the system becomes attractive as observed by enhanced scattering in the low $Q$ region. This attraction is modeled by short range Baxter's sticky hard sphere potential. As reported earlier the width of the attractive well is kept fixed and equal to $3 \AA$ typically that of the size of the water molecule [6].

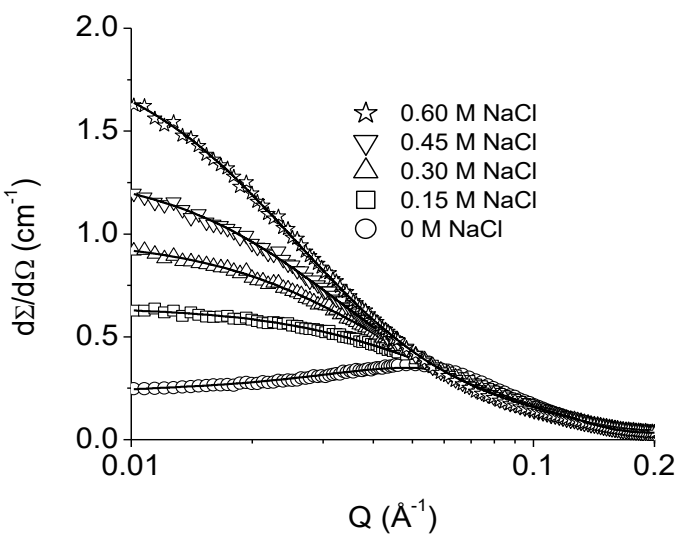

FIGURE 1. SANS data of 5 wt $\%$ lysozyme solution with varying concentration of $\mathrm{NaCl}$.

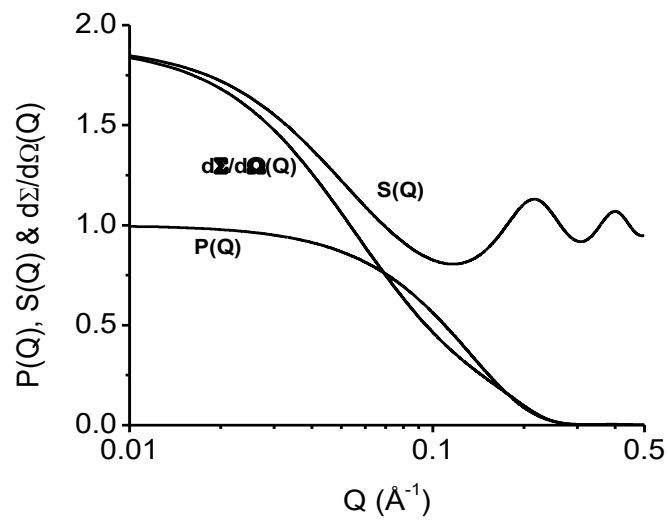

FIGURE 2. The calculated $P(Q), S(Q)$ and $d \Sigma / d \Omega$ curves for Baxter's sticky hard sphere potential.

Figure 2 shows typical form factor, structure factor and scattering cross section for a lysozyme solution calculated using Baxter's sticky hard sphere potential. It is clear that the build-up of the scattering in the low $Q$ region is because of $S(Q)$ where as the scattering at high $\mathrm{Q}$ is decided by the $\mathrm{P}(\mathrm{Q})$. The fitted parameters of the analysis of the $5 \mathrm{wt} \%$ protein solutions using equation 1 are given in table 1 . The depth of the attractive potential is found to increase with $\mathrm{NaCl}$ concentration. On the other hand, there is no change observed in the structure of protein molecules. It is found that when the attractive potential is found to be significantly larger $\left(-2.37 \mathrm{k}_{\mathrm{B}} \mathrm{T}\right)$ than the thermal energy, protein solution undergoes liquid-liquid phase transition. The schematic of this phase transition is shown in figure 3 . The increased attraction between protein molecules (>0.6 M NaCl) leads to clustering of protein molecules and these clusters further aggregate to form gel.

TABLE 1. Fitted parameters of $5 \mathrm{wt} \%$ aqueous lysozyme solution on $\mathrm{NaCl}$ addition.

\begin{tabular}{|c|c|c|c|}
\hline \multirow{2}{*}{$\begin{array}{c}{[\mathrm{NaCl}]} \\
(\mathrm{M})\end{array}$} & \multicolumn{2}{|c|}{ Protein Dimensions } & \multirow{2}{*}{$\begin{array}{l}\text { Interaction } \\
\text { Potential } \\
\left(\mathrm{U} 0 / \mathrm{k}_{\mathrm{B}} \mathrm{T}\right)\end{array}$} \\
\hline & $\begin{array}{l}\text { Semimajor } \\
\text { axis }\end{array}$ & $\begin{array}{l}\text { Semiminor } \\
\text { axis }\end{array}$ & \\
\hline 0.0 & $22.0 \AA$ & $13.5 \AA$ & - \\
\hline 0.15 & $22.0 \AA$ & $13.5 \AA$ & -1.63 \\
\hline 0.30 & $22.0 \AA$ & $13.5 \AA$ & -2.11 \\
\hline 0.45 & $22.0 \AA$ & $13.5 \AA$ & -2.28 \\
\hline 0.60 & $22.0 \AA$ & $13.5 \AA$ & -2.37 \\
\hline \multicolumn{2}{|c|}{$\begin{array}{l}0.000 \\
0 \% 0 \% \\
00 \% \\
000 \\
0\end{array}$} & \& & \\
\hline \multicolumn{2}{|c|}{ monomers } & usters & gel \\
\hline
\end{tabular}

FIGURE 3. Schematic of liquid-liquid phase transition in protein solution on addition of salt resulting in gel formation.

To conclude, SANS studies show that the salt induced protein gelation is governed by the interactional changes undergoing in the protein solutions instead of the continuous aggregation of the protein molecules.

\section{REFERENCES}

1. A. Stradner, H. Segdwick, F. Cardinaux, W. Poon, and P. Schurtenberger, Nature 432, 492-495 (2004).

2. Y. Liu, E. Fratini, P. Baglioni, W.R. Chen and S.H. Chen, Phy. Rev. Lett. 95, 118102 (2005).

3. D.I. Svergun and M.H.J. Koch, Rep. Prog. Phy. 66, $1735-1782$ (2003).

4. S.V.G. Menon, C. Manohar, and K.S. Rao, J. Chem. Phys. 95, 9186-9190 (1991).

5. S. Chodankar and V.K. Aswal, Phy. Rev. E 72, 041931 (2005)

6. P.S. Goyal, S.V.G. Menon, B.A. Dasannacharya and P. Thiyagarajan, Phy. Rev. E 51, 2308-2315 (1995). 